\title{
Halbleiter-basierte Hochleistungsgeneratoren für Breitbandpuls-Abstrahlung
}

\author{
T. Ehlen \\ Fachhochschule-Gelsenkirchen, Neidenburger Str. 10, 45877 Gelsenkirchen, Germany
}

Zusammenfassung. Irradiated DS pulses beneath HPM and UWB are capable of disturbing the signal processing or even destroying the semiconductor structure. This paper presents the History of DS sources and their physical and electronic working principle, their technology and the potential application fields

\section{Einleitung}

Elektromagnetische Pulse, die mit hoher Feldstärke auf elektronische Systeme treffen, sind in der Lage, deren Funktionalität kurzfristig oder nachhaltig zu beeinflussen. Im letzten Jahrzehnt wurde die Wirkung von schmalbandigen CW und HPM-Pulsen, den ultrabreitbandigen UWB-Pulsen und den breitbandigen DS-Pulsen eingehend untersucht (Bohl et al., 1998, Ehlen et al., 1998). Alle Signalformen können dabei digitale und analoge Signale stören und elektronische Komponenten zerstören. Die Kenntnis dieser Effekte bildet die Grundlage für notwendige anzuwendende Härtungsmaßnahmen und ermöglicht, für neue Technologien Voraussagen bezüglich ihrer elektromagnetischen Empfindlichkeit zu treffen. Im militärische Bereich wurden die Quellen auf maximale Störfähigkeit feindlicher Elektronik optimiert. Dieser Prozess hält noch an. Zielspektren bilden dabei die Beeinflussung der Flugbahn von gegnerischen Flugkörpern und Fliegerfäusten, die vollständige Unterdrückung funkund drahtgebundener Kommunikation, die Zerstörung von Abhör- und Überwachungseinrichtungen, oder der Konvoischutz durch vorzeitige Auslösung von Minen. Im zivilen Bereich wird die gezielte Störbeeinflussung der Motorsteue-

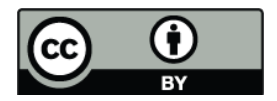

Correspondence to: $\mathrm{T}$. Ehlen (tilo.ehlen@fh-gelsenkirchen.de) rung bei Kraftfahrzeugen (elektronische Strassensperre) und Schnellbooten (Schmuggel) ausgenutzt. Ebenso werden geeignete abgestrahlte elektromagnetische Pulse bereits heute eingesetzt, um die Daten und die Zugriffselektronik von elektronischen Speichern (USB-Stick, Festplatte) oder RFIDChips bewusst zu zerstören und damit die personenbezogenen Informationen (z.B. auf dem neuen Personalausweis) permanent zu löschen.

Grundlage für die Beurteilung der Signaleffektivität bilden Empfindlichkeitsuntersuchungen an Kraft-fahrzeugen, PC's und Mikrokontrollern, SPS-Steuerungen, Monitoren und vielen militärischen Geräten.

Als effektive Signalformen haben sich neben $\mathrm{CW}$ auch gepulste breitbandige Signale erwiesen (Bohl et al., 2001). Konventionelle Breitband-Generatoren arbeiten mit Hochspannungs-Überschlagsschaltern, was jedoch Einschränkungen in Bezug auf Triggerbarkeit und Lebensdauer mit sich bringt. Im folgenden werden diverse alternative Breitbandpulser Realisierungen im Kilovolt Bereich und Antennenkopplungen vorgestellt von Avalanche- und Steprecovery-basierten Diodenschaltern bis hin zu nichtlinearen magnetischen Pulsaufsteilern.

Neben HPM (High Power Microwave) Pulsen, modulierter CW, und UWB (Ehlen, 2007) haben sich in den letzten Jahren DS-Signale als besonders effektiv bezüglich Elektronikstör- und zerstörvermögen erwiesen.

DS-Pulse (Damped Sinus=gedämpfter Sinus) bestehen aus einer abklingenden Sinusschwingung mit Frequenzen zwischen $50 \mathrm{MHz}$ und $700 \mathrm{MHz}$, Pulslängen von um $10 \mathrm{~ns}$ bzw. zehn Schwingungszyklen, Feldstärken von vielen hundert Kilovolt/m und Pulswiederholraten bis ca. 100 Hz. Das Spektrum besitzt eine Bandbreite von ca. 20\%. DS-Pulse regen typischerweise die kleinsten Resonanzfrequenzen der Zielsysteme an und besitzen daher eine besonders gute

Published by Copernicus Publications on behalf of the URSI Landesausschuss in der Bundesrepublik Deutschland e.V. 


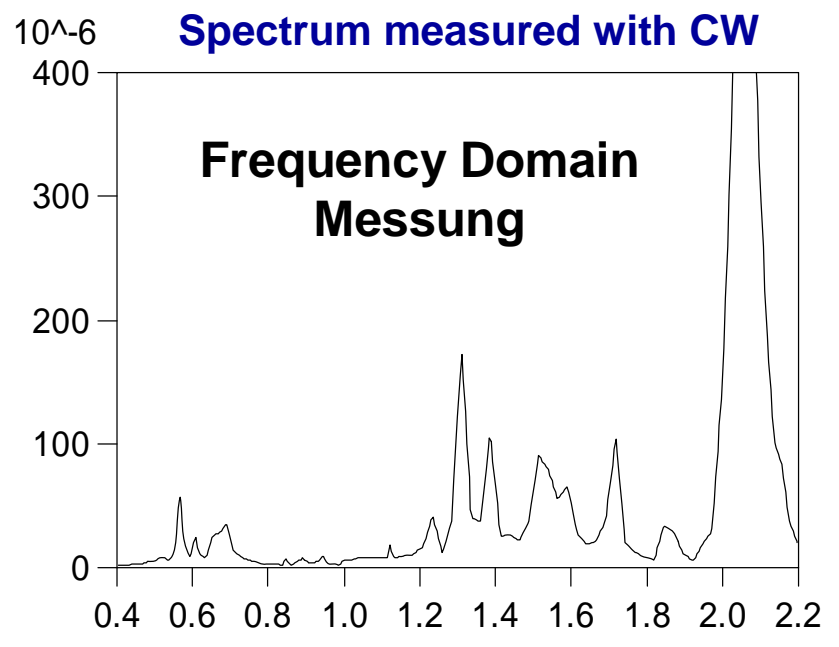

$10^{\wedge} 9$

Abb. 1. Typisches Empfindlichkeitsspektrum eines medium geschirmten elektronischen Systems.

Koppeleffektivität. Elektronikstörungen wie auch Zerstörungen insbesondere bei digitalen Systemen werden selbst bei medium-geschirmten Systemen erreicht. Die Störung bei analogen Systemen wird durch die geeignete Wahl der Pulswiederholrate dominiert.

\section{Historie}

Bereits 1887 wurde von Heinrich Hertz zum Nachweis der Abstrahlung elektromagnetischer Wellen ein erstes DSSystem verwendet. Mit einer Ladespannung von einigen Kilovolt wurde die Kapazität eines Dipols langsam über einen Widerstand R aufgeladen mittels eines Funkeninduktors. Parallel zur Dipolkapazität befand sich an den beiden zueinander zeigenden Enden der beiden Dipolschenkel eine Funkenstrecke, die beim Erreichen der kritischen Spannung durchzündete und einen resonanten Strom auf den Dipolschenkeln erzeugte. Damit wurde eine gedämpfte Sinusschwingung abgestrahlt. Die Induktivitäten $L$ in den beiden Zuleitungen trennten die HF von der Ladeeinheit.

1896 stellte Marconi den auf dem gleichen Sendeprinzip arbeitenden DS-Generator mit Empfänger als erstes "drahtloses Telegrafiesystem mit gedämpften Wellen" (Morsen) vor mit einer HF-Leistung von 10-20 Watt und einer Funkenspannung von ca. $80 \mathrm{kV}$. Die Frequenz war für diese Anwendung bereits auf $2.5 \mathrm{MHz}$ reduziert (see http://www. seefunknetz.de/homepage.htm).

Zur Erhöhung der abgestrahlten Feldamplitude wurde folgend von Braun die Antenne induktiv an die Funkenstrecke gekoppelt. Damit lag nur noch die HF an der Antenne an und zudem war geringere Funkendurchbruchspannung notwendig. Es trat dabei nun ein modifizierter DS-Puls auf, der

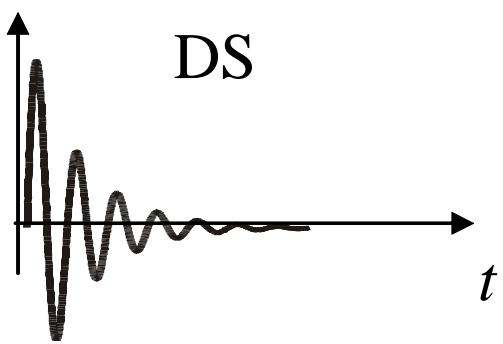

Abb. 2. Typische DS-Signalform im Zeitbereich.

durch die Schwebungsfrequenz aus Primärresonanzfrequenz und Sekundärkreisresonanz geprägt wurde. Diese Knallfunkensender besaßen jedoch eine geringe Pulswiderholrate von ca. $20 \mathrm{~Hz}-100 \mathrm{~Hz}$. Die von Max Wien 1906 modifizierte Funkenstrecke zur Löschfunkenstrecke vermied ein wiederholtes Zünden nach der ersten Schwingungshalbperiode. Während sich bei einem Knallfunkensender die Schwingungsenergie des Primärkreises nur etwa zu 20\% in HF-Abstrahlung umsetzt, sind es bei diesen so genannten Löschfunkensendern $50 \%$ bis $70 \%$. Die abgestrahlte Signalform besaß nun wieder eine typische jedoch schmalbandigere DS-Form und die Pulswiderholrate konnte auf $500 \mathrm{~Hz}$ gesteigert werden. Marconi erhöhte die Pulswiederholrate durch eine Anordnung von rotierenden Funkenstrecken (Abb. 5).

In den 1920er Jahren wurden die Knallfunkensender verboten, da sie durch die große Bandbreite der abgestrahlten DS-Einzelpulse den Empfang anderer Sender störten.

\section{Heutige ds-Quellen}

Während bei UWB-Pulsern die Flanke eines schnellen Hochspannungspulses unmittelbar über eine differenzierend wirkende Antenne (TEM-Horn, IRA) abgestrahlt wird, wird bei heutigen DS-Systemen (Abb. 6) eine Pulsformungsleitung (PFL) als Energiezwischenspeicher langsam über eine DC-Spannungsquelle auf viele hundert $\mathrm{kV}$ aufgeladen. Am einen Ende der Leitung befindet sich eine fehlangepasste Breitbandantenne, am anderen Ende eine Funkenstrecke. Nach Zünden der als schließenden Schalter wirkende Funkenstrecke wird die erzeugte Pulsflanke zwischen den beiden Leitungsenden hin- und herreflektiert. Der dabei entstehende charakteristische DS-Impuls wird teilweise auf die Breitbandantenne gekoppelt und abgestrahlt.

Als Antenne fungieren Konus, Dipol oder TEM-Hörner. Wie bei den ersten Versuchen von Hertz kann die abgestrahlte Feldstärke durch Reflektoranordnungen erhöht werden,

Verzichtet man auf die PFL und verwendet die Eigenkapazität der Antenne als Energiespeicher, so definiert - wie bei dem ursprünglichen Hertz'schen DSGenerator - die Reflektion an den Antennenunstetigkeiten die DS-Ringingfrequenz, wie dies in Abb. 7 links mit einer planaren Vivaldi-Antenne der FH-Gelsenkirchen zu sehen ist. 

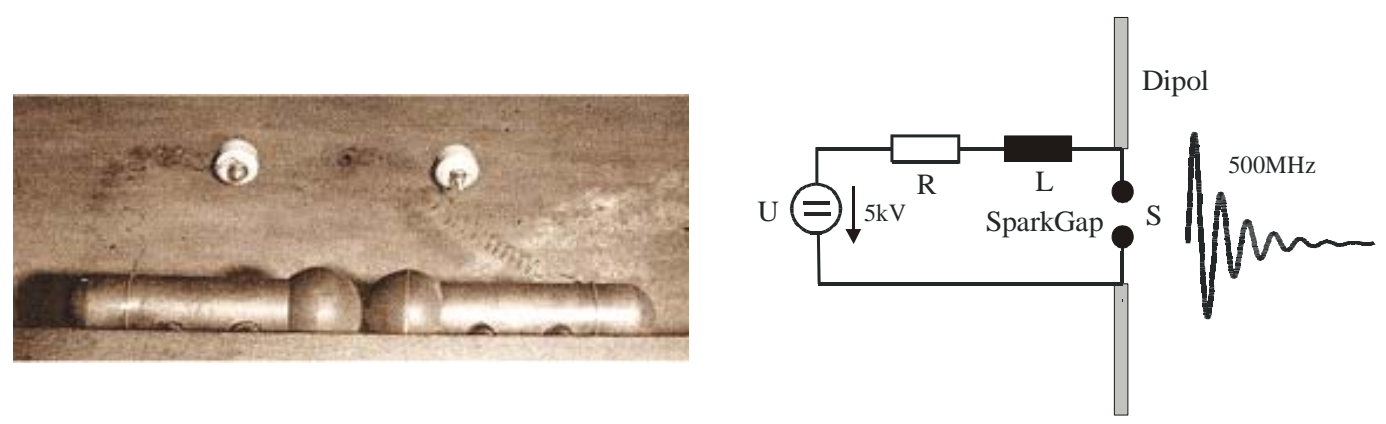

Abb. 3. Von Hertz verwendeter Sende-Dipol mit Funkenstrecke.

Die Hochspannungsgenerierung basiert klassisch auf einem Marx-Generator oder Teslatransformator mit Spannungen bis ca. $2 \mathrm{MV}$. Die bisherigen als Schalter fungierenden schnellen Überschlagsstrecken beschränken die Pulswiederholrate auf einige $100 \mathrm{~Hz}$ mit Schaltgeschwindigkeiten um $100 \mathrm{ps}$.

DS-Systeme zeigen trotz hoher Spannungen sehr kompakte Bauformen. Einzelsysteme können ebenfalls zu Arrays kombiniert werden, wobei wegen der geringeren Anstiegszeit auch die Triggerzeittoleranzen im Vergleich zu UWB unkritischer sind. Die Fa. Diehl/Röthenbach bietet neben autonomen Koffer DS-Systemen auch kaskadierte Vielfach-DS-Arrays mit Feldstärken über $500 \mathrm{kV} / \mathrm{m}$ als Testsysteme an.

\section{Halbleiterquellen}

Ersetzt man die aufgeladene PFL und den schließenden Schalter $(1-\varepsilon(t))$ - also den Ausschaltpuls - durch eine nicht geladenen PFL mit Sprunganregung $\varepsilon(t)$ - also durch einen Einschaltpuls, so wird der inverse DS-Puls abgestrahlt. Technisch bedeutet das die unmittelbare Verbindung eines schnell schaltenden Pulsgenerators (schnelle steigende oder fallende Flanke) mit der PFL oder der Antenne.

Der Trend zu höheren Pulswiderholrate $>1 \mathrm{kHz}$, exakterer Triggerbarkeit und erhöhter Wartungsfreiheit verlangt, die Funkenstrecken durch schließende Halbleiterschalter zu ersetzen oder mittels schnellen Halbleiterpulsern die PFL unmittelbar zu erregen. Für Schwingfrequenzerregung bis $500 \mathrm{MHz}$ sind typischerweise Schaltflanken $<0.5$ ns notwendig. Zur Erzeugung dieser schnellen Flanken in Verbindung mit der zu schaltenden Spannung bei vielen duzenden Kilovolt sind nur einige wenige Halbleiterschaltungskonzepte in den Pulsern zu finden.

Neben den langsameren Thyristoren und GTOs sind insbesondere CMOS-Schalter und IGBT's in der Lage, geeignet schnelle Schaltflanken zu liefern - die Schaltspannungen der Einzelbauteile sind dabei bereits um $500 \mathrm{~V}-1 \mathrm{kV}$ ausgelegt. Je höher die maximale Schaltspannung, desto größer
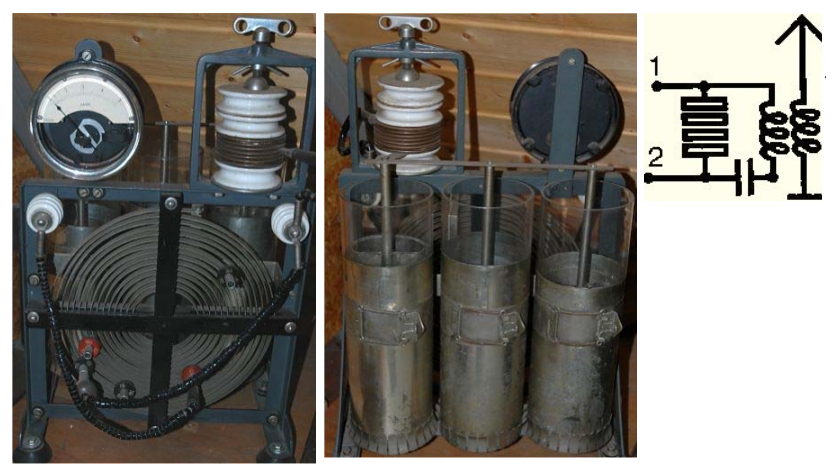

Abb. 4. DS-Sender (Lösch funkensender) TK05 von Telefunken mit $500 \mathrm{~Hz}$-Reprate. Links Ansicht von vorn mit variabler Selbstinduktion, Amperemeter und Löschfunkenstrecke, mitte: Ansicht von hinten mit den drei Leydener Flaschen des Stoßkreises, rechts: Schaltbild mit Löschfunkenstrecke und Kondensator im Primärkreis (Stoßkreis).

der $R_{\mathrm{DS} o n}$-Widerstand bei den MOS-Varianten. Um einen Serienwiderstand unter $1 \mathrm{Ohm}$ zu erreichen, werden daher häufig 4 oder 6 Halbleiter parallel geführt. Mit zunehmender Schaltspannung vergrößert sich auch die Bauform und erhöht aufgrund der zunehmenden parasitären Induktivitäten die Schaltzeit auf mehrere Nanosekunden. Zudem muss das Gate $(1 \mathrm{nF})$ schnell genug mit hohem Strom angesteuert werden, was einen zusätzlichen Treiber erfordert. Da der Schalter durch die DS-Schwingungsform auch negative Ströme bedingt, müssen bei den IGBT's externe Freilaufdioden angebracht werden - bei den CMOS-Schaltern sind diese typischerweise integriert. Selbst bei maximaler Schaltspannung von bisher $4 \mathrm{kV}$ erfordern technisch interessante Anwendungen von $>20 \mathrm{kV}-100 \mathrm{kV}$ eine Serienschaltung vieler dieser Schalter. Zur Symmetrierung der Sperrspannung werden wie bei Audioverstärkern hochohmige Widerstände parallel zur Drain-Source oder Kollektor-Emitter-Strecke verwendet. Die quasi gleichzeitige Triggerung aller Schalter erfolgt in Baker and Johnson, 1992 z.B. kapazitiv/resistiv. Zur Vermeidung 

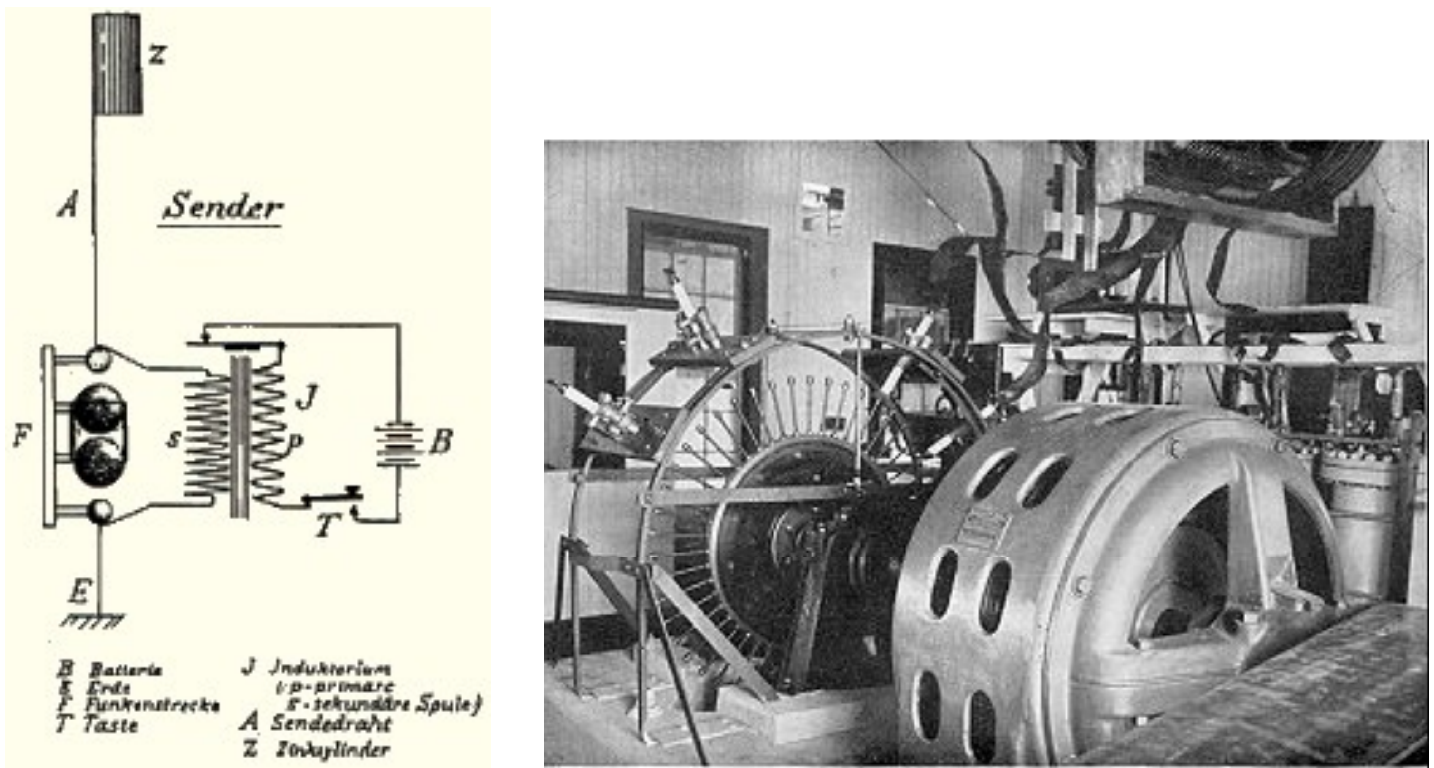

Abb. 5. Links DS-Sender (Knallfunkensender) von Marconi 1897 ,rechts: rotierende Funkenstrecke von Marconi zur Erhöhung der Pulswiderholrate in einem Telegrafiesender von 1908 mit $20 \mathrm{~kW}$ HF-Leistung. Quelle: http://www.seefunknetz.de.
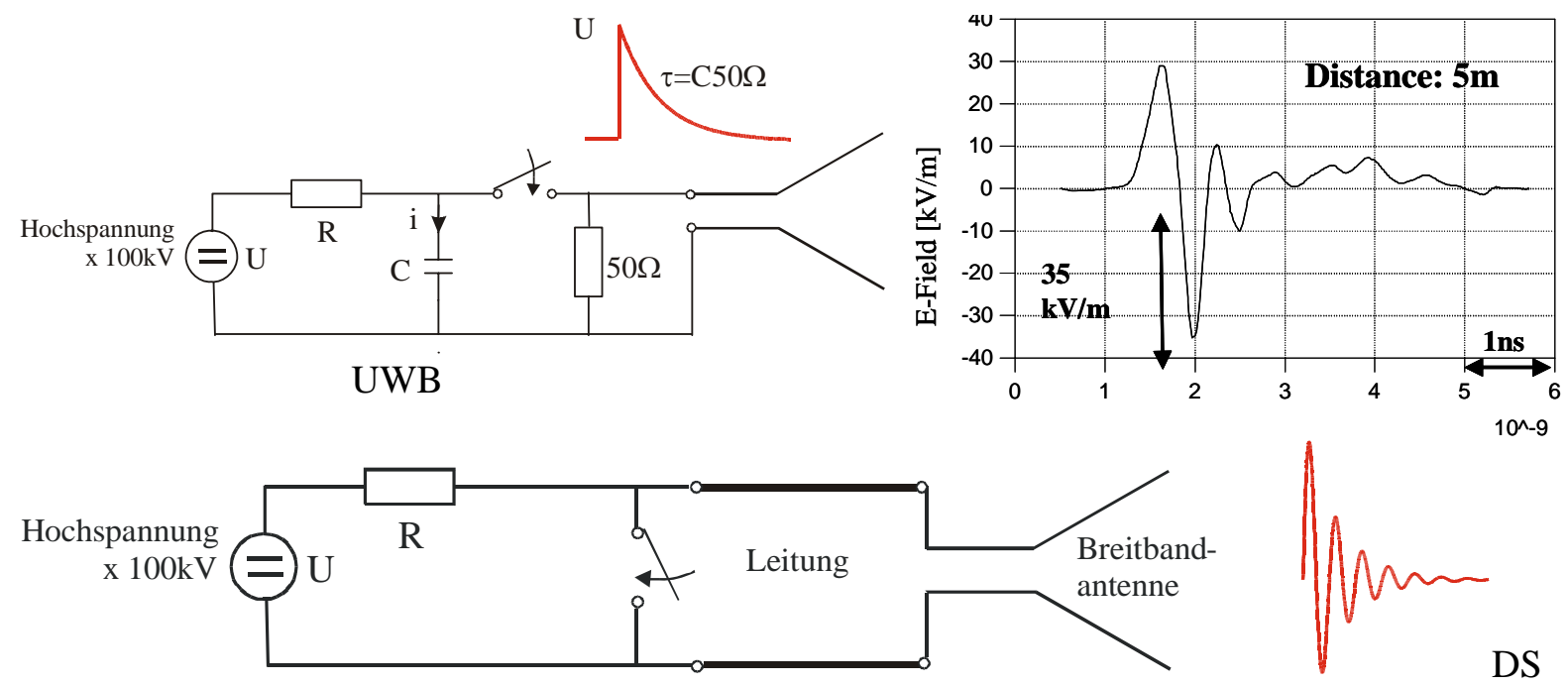

Abb. 6. Oben: UWB-Erzeugungsprinzip, unten: heutiges DS-Erzeugungsprinzip.

von Hochspannungsspitzen am Gate und induktiv gekoppelter Gatestörimpulsen beim Zünden findet man insbesondere bei Schaltern russischer Systeme die EMV-gerechtere transformatorische Triggerung.

Von Grekhov/Mesyats Mesyats, 2005 sind die "magnetischen Schalter" in schnellen HochspannungsPulsgeneratoren bekannt. Das Grundprinzip basiert auf der Resonanzüberhöhung eines auf VCC geladenen Kondensators $\mathrm{C} 2$ der gemäß Abb. 11 über die Induktivität L1 auf eine kleine Kapazität C3 umgeladen wird. Die dabei entstehende Spannungsspitze an C3 ist doppelt so hoch wie die Versorgungsspannung. Der ursprüngliche Schaltprozess wird zunächst durch einen im Mikrosekundenbereich schaltenden Thyristor hervorgerufen. Diesen Prozess der Spannungsverdopplung lässt sich nun durch zeitgerechtes Hinzuschalten eines weiteren LC-Kreises mit viel höherer Resonanzfrequenz erweitern. Damit erzielt man eine weitere Spannungsverdopplung auf $4 \times \mathrm{VCC}$ in der ausgelösten 

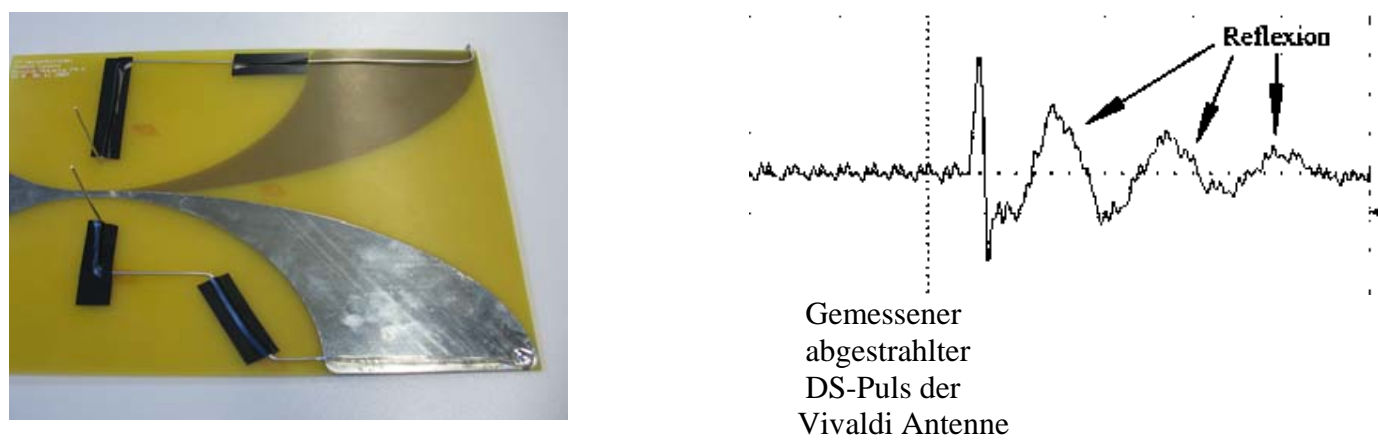

Abb. 7. Links: 5 kV-Vivaldiantenne der FH-Gelsenkirchen und rechts: abgestrahlter DS-Puls mit UWB-Anteil.

Schwingung. Die Choke-Induktivitäten L2 und L4 dienen lediglich der DC-Aufladung der Kondensatoren C2 und C4. Der schließende Schalter zusammen mit der Induktivität L5 der zweiten Stufe wird technisch durch eine nichtlineare Induktivität ersetzt. Bei kleinen Strömen besitzt diese sehr große Induktivitätswerte und wirkt quasi wie ein Leerlauf. Bei geeigneter Dimensionierung der Windungszahl, des Querschnitts und des magnetischen Kernmaterials geht die Spule ab einer kritischen Stromstärke in die Sättigung der Induktivitätswert nimmt rapide ab und löst die zweite hochfrequente Oszillation aus. Neben der Pulsamplitudenerhöhung hat zudem eine Pulsversteilerung stattgefunden. Mehrere nichtlineare magnetische Pulskompressionsstufen ermöglichen in realisierten Pulsern des Ioffe-Instituts Pulsausgangsspannungen von über $200 \mathrm{kV}$ bis in den Megavoltbereich (Mesyats and Rukin, 2000). Modifikationen weisen zusätzliche Spannungstransformation mittels nichtlinearer Transformatoren auf.

Die Pulsflanken dieser Pulser liegen im einstelligen Nanosekundenbereich. Zur weiteren Aufsteilung werden die Ladungsträgerspeichereffekte von Varaktordioden, PINDioden und Steprecovery-Dioden eingesetzt.

Wird eine sinusförmige Spannung an eine Diode gelegt, so fließt ein positiver Strom in die Diode. Die dabei in der Diode gespeicherten Ladungsträger müssen bei der negativen Spannungswelle wieder ausgeräumt werden und erzeugen einen negativen Diodenstrom, der wenn alle Ladungsträger ausgeräumt sind, sehr schnell ausschaltet. Die Strompulsflanke kann im Subnanosekundenbereich liegen. Typischerweise wird der Diodenausschaltstrom über eine Induktivität geführt, so dass eine dort induzierte Spannung - die an den Verbraucher gelangt, ein Vielfaches der anregenden Spannungsamplitude besitzt. Einige russische Varianten (Grekhov et al., 1994) wurden auf besonders hohe Stromdichten, schnelles Schaltverhalten und ein asymmetrisches Stromverhalten getrimmt, so dass der Stromabriss bereits auftritt, wenn noch nicht alle gespeicherten Ladungsträger ausgeräumt sind. Kombiniert mit dem Magnetischen SchalterSystem als Erreger, sind z.B. Pulser bis $1 \mathrm{MV} / 50 \Omega$ am High

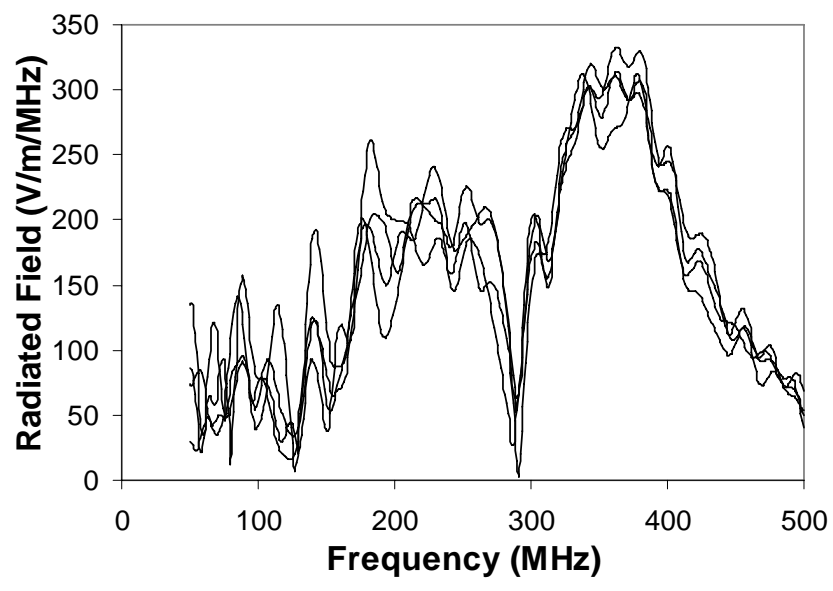

Abb. 8. Spektrum des abgestrahlten DS-Pulses eines 3er-Arrays der Fa. Diehl.

Current Institut in Tomsk entwickelt worden. Im Gegensatz zu den von Hewlett Packard angebotenen Step-RecoveryDioden im Gigahertzbereich zur Erzeugung von $<100$ ps sind die russischen Dioden für hohe Spannungen $(1 \mathrm{kV})$ und Ströme bis (1 kA) ausgelegt. Die pillenartigen Dioden lassen sich zu Stacks zusammenfassen - viele hundert in Serie, um die $\times 100 \mathrm{kV}$-Pulse zu widerstehen und mehrere Stacks parallel, um die Stromtragfähigkeit zu erhöhen.

Hybride Varianten (High current Institut, Ekaterinburg) erzeugen die Hochspannung zwar mit speziellen SRDHalbleitern, steilen jedoch die Pulsflanken mit nachgeschalteten Sparc-Gaps auf. Alternativ werden zur weiteren Pulsflankenaufsteilung in den Ausgangskreis Avalanchedioden eingebaut, die für Spannungen von duzenden von Kilovolt und Anstiegszeiten um 100 ps die schnellsten Halbleiterpulser zieren. 

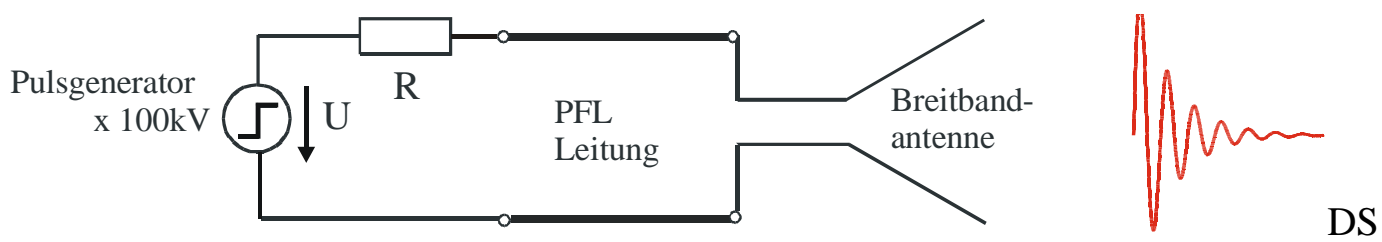

Abb. 9. DS-Erzeugung mit Pulsgenerator.

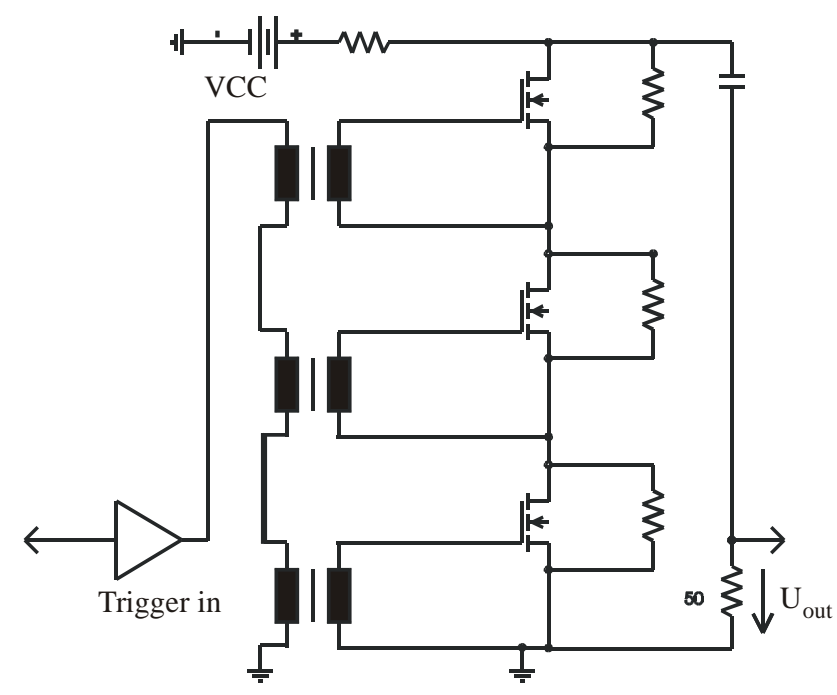

Abb. 10. Transformatorische Triggerung eines $2 \times 3$ CMOSSchalters zur DS-Pulsanregung mit Symmetrierwiderständen.

Während beim statischen Avalanche-Effekt wie bei den Z-Dioden eine konstante Z-Spannung am Halbleiterbauelement anliegt, bricht diese Spannung bei Anlegen einer sehr schnellen Überspannung vollständig zusammen (dynamischer Avalanche-Effekt) - das Bauteil zeigt dann einen Kurzschluss. Wie in Abb. 13 werden in den Pulsern die Dioden statisch bis kurz vor ihrer statischen Durchbruchspannung vorgespannt. Ein Triggerpuls entlädt CL1. Die dabei entstehende Überspannung an D1 initiiert dann den vollständigen Spannungszusammenbruch auf 0V an D1. Wegen CL2 «CL1 entlädt sich CL2 quasi vollständig und startet den Durchbruch von D2 usw. Der letzte 4kV-Entladepuls wird kapazitiv an die $50 \mathrm{Ohm}$ Last weitergegeben. Durch Kaskadierung mehrerer solcher Stufen steilt sich der Ausgangspuls stetig auf (bis ca. $100 \mathrm{ps}$ ). Die optimierten russischen Avalanche-Dioden zeichnen sich durch eine extrem geringe Sperrschichtkapazität und eine besonders hohe $\mathrm{Fe}$ stigkeit bezüglich gepulster Hochspannung aus (z.B. $20 \mathrm{kV}$ je Diode), was die Stufenanzahl extrem reduziert.

Die UWB-Pulser der Fa. Kentech verwenden kaskadierte 200 V-400 V Avalanche-Transistoren, womit bei einer Stufenzahl von 10 etwa ein $2 \mathrm{kV}$-Puls gewonnen wird. Man kombiniert dann viele dieser Einzelpulser über koaxiale Leitungstransformatoren auf Ausgangsspannungen von über $100 \mathrm{kV}$. An der FH-Gelsenkirchen wurde ein koaxialer $5.5 \mathrm{kV}$-Subnanosekunden DS/UWB-Pulser entwickelt (Ehlen, 2005), der den transienten Avalanche-Effekt, wie bei Trapatt-Dioden, in herkömmlichen Dioden und Transistoren ausnutzt (Abb. 13). Halbleiterpulser mit sehr hohen Pulswiederholraten von über $1 \mathrm{MHz}$ arbeiten allerdings mit Spannungen von nur einigen Kilovolt. Man schaltet daher viele dieser Pulser mit jeweils eigener Antenne zu einem Array zusammen. Das amerikanische BAS-Array triggert viele duzend Einzelsysteme über Lichtpulse.

Weitere Forschungsarbeiten beschäftigen sich mit der Aufsteilung von Pulsflanken durch nichtlineare Leitungen. Hierzu werden Pulse im Nanosekundenbereich, die noch keine DS-Pulse erregen können, über eine Pulsflankenversteilerung für den Subnanosekundenbereich aufbereitet.

Die Wirkungsweise der Flankenaufsteilung beruht auf den unterschiedlichen Ausbreitungsgeschwindigkeiten der Spannungsamplituden. Diese zeigen wie z.B. bei der Ausbreitung von akustischen Wellen eine Amplitudenabhängigkeit der Ausbreitungsgeschwindigkeit. Bewegen sich größere Spannungsamplituden schneller auf der Leitung als kleine Spannungsamplituden, so holen die großen Spannungswerte die kleinen ein und eine abfallende Flanke steilt sich auf, eine ansteigende Flanke flacht sich ab.

Die verlustlose Leitung wirkt wie eine Kaskade von vielen Längsinduktivitäten und Querkapazitäten. Mit $L^{\prime}$ (Induktivität pro Meter) und $C^{\prime}$ (Kapazität pro Meter) berechnet sich die Ausbreitungsgeschwindigkeit:

Ausbreitungsgeschwindigkeit : $v=\frac{1}{\sqrt{L^{\prime} C^{\prime}}}$

Sorgt man dafür, dass entweder die Induktivität oder die Kapazität von der Spannungsamplitude abhängt, so erreicht man solch eine amplitudenabhängige Ausbreitungsgeschwindigkeit. Zur Realisierung werden entweder die Querkapazitäten durch sperrende Dioden (Diffusionskapazität verringert sich mit zunehmender Spannung) oder die Längsinduktivitäten durch nichtlineare in Sättigung getriebene Induktivitäten ersetzt (Induktivität verringert sich mit zunehmendem Strom). Die technische Begrenzung liegt beim Einsatz der Dioden an der begrenzten Sperrspannung - Spannungsamplituden bis ca. $3.5 \mathrm{kV}$ wurden hier bisher 

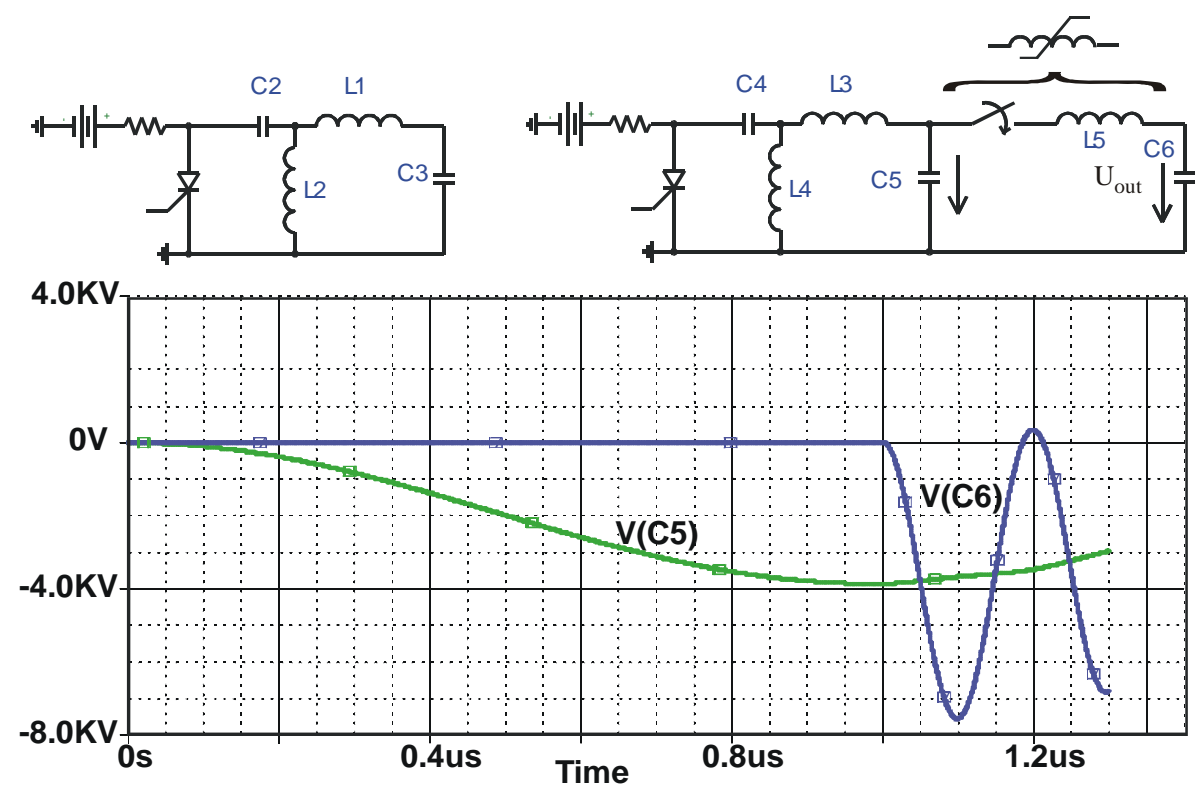

Abb. 11. Prinzip der Resonanzüberhöhung und Pulskompression mit Thyristor; rechts oben: Kaskadierte Pulskompression - gesättigte Induktivität repräsentiert schließenden Schalter; unten: Spannungsverläufe bei 2 kV VCC.
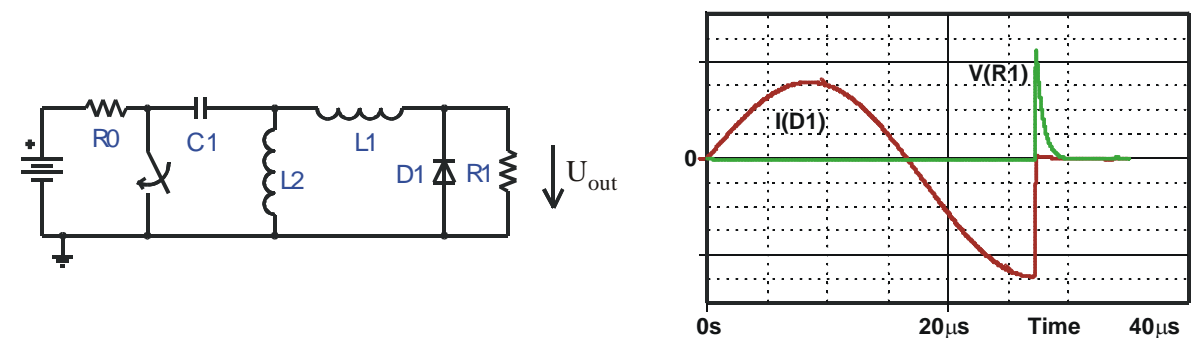

Abb. 12. Ansteuerung SOS-Pulser mit Diodenstrom und pulsförmiger Ausgangsspannung an R1.

erreicht (Baker, 1993). Von Anwendungen mit nichtlinearen Induktivitäten werden bis $90 \mathrm{kV}$ Spannungsamplituden in Kataev and Lipatov, 1971 berichtet oder bis zu $100 \mathrm{kV}$ mit 86 ps Flankensteilheit in Seddon et al., 2007. Sie leiden jedoch an der Reproduzierbarkeit der Materialien und der Temperaturdrift der magnetischen Kennlinie.

\section{Literatur}

Bohl, J., Ehlen, T., and Sonnemann, F.: HPM-Effects in LFElectronic-Circuits: A Numerical Approach, EUROEM98, TelAviv, Israel, June 1998.

Ehlen, T., Bohl, J., and Sonnemann, F.: Electromagnetic Coupling Paths to Electronic Systems Connected with Electronic Setups and Destruction Mechanism, IST/SET Symposium 98, Aalborg, Denmark, October 1998.

Bohl, J., Ehlen, T., Staines, G., and Sonnemann, F.: Interference and Destruktion Mechanisms of Electronic Irradiated with HPM and
UWB Sources, ICEAA01, Turin, Italy, September 2001.

Ehlen, T. and Bohl, J.: Einführung in Chaos und Nichtlinearitäten bezüglich Elektromagnetischer Effekte, BakWVT-Mannheim EME-Symposium, 2005.

Taylor, D. and Giri, D. V.: High-Power Microwave Systems and Effects, Taylor\&Francis, 1994.

Benford, J.: Swegle, High-Power Microwaves, Artech House, 1992.

Ehlen, T., Bohl, J., Kuhnke, R., and Sonnemann, F.: Compact HPM and UWB Sources using Explosives - The potential of Future non lethal Warhead Systems, EUROEM 2000, Edinburgh, June 2000.

Ehlen, T.: UWB-Halbleiterpulser - Konzepte und Realisierungen mit COTS-Bauteilen BakWVT-Mannheim EME-Symposium, 2005.

Ehlen, T.: Bewusste Elektronikstörung durch gestrahlte HPM, UWB und DS-Pulse, EEEfCom 2007, Ulm, Germany, Juni 2007.

Baker, R. J. and Johnson, B. P.: Series operation of power MOSFETs for high speed high voltage switching applications, Department of Electrical Engineering, University of Nevada, 

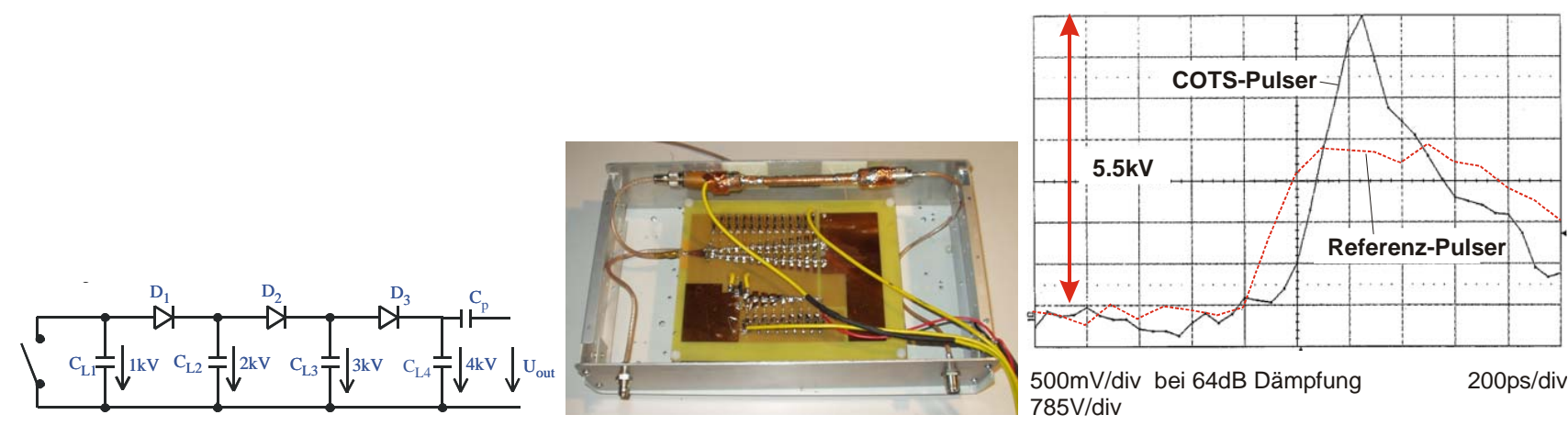

Abb. 13. 3-stufiger UWB-Pulser der FH-Gelsenkirchen mit COTS-Dioden im transienten Avalanche Betrieb und koaxialer Endstufe (COTS=commercial of the shelf).

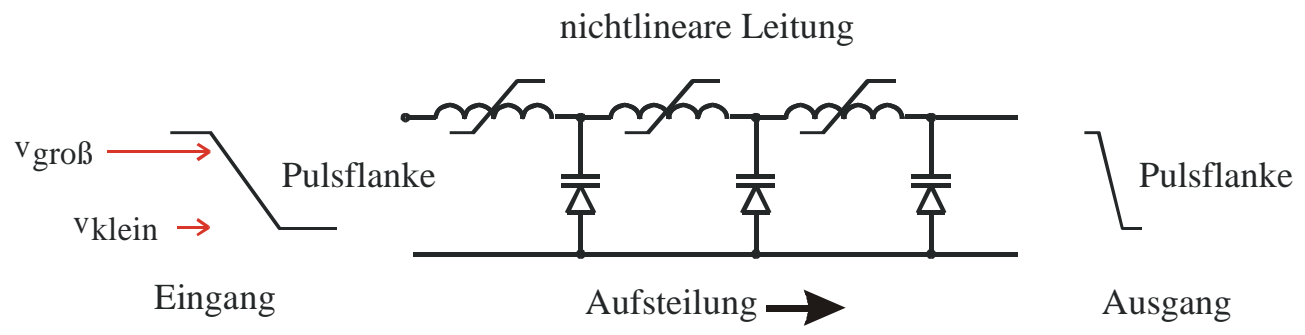

Abb. 14. Prinzip der nichtlinearen Leitung zur Aufsteilung der Pulsflanken.

Reno, 1992.

Mesyats, G. A.: Pulsed Power, Springer-Verlag, 2005.

Mesyats, G. A. and Rukin, S. N.: $1 \mathrm{MV}, 500 \mathrm{~Hz}$, all solid state nanosecond driver for streamer corona discharge, ieee conf. on high power particle beams, agona, japan, 192 pp., 2000.

Grekhov, I. V., Efimov, V. M., Kardo-Sysoev, A. F., and Korotkov, S. V.: RF Patent No. 2009611, 1994.

Seddon, N., Spikings, C. R., and Dolan, J. E.: RF Pulse Formation in Nonlinear Transmission Lines, Pulsed Power Plasma Science, PPPS-2007, Digest of Technical Papers, no. 153, 2007.

Kataev, I. G. and Lipatov, N. F.: Generator of high power nanosecond pulses on non-linear transmission lines with ferrite,Instruments and technology of experiments, N5, 1971.
Korchuganov, V., Matveev, Y., and Shvedov, D.: Ferrite line to decrease rise time of high-voltage nanosecond pulses, Proceedings of the 2001 Particle Accelerator Conference, 2001, PAC 2001, 5, 4047-4049, 18-22 June 2001.

Brooker, C., Altieri, N.,, Eastwood, G., Hoad, R., and Dolan, J. E.: " $90 \mathrm{kV}, 1.8 \mathrm{kA} 85 \mathrm{ps}$ risetime electromagnetic shock line for UWB application, Electronics Letters, 35(25), 2210-2212, 9 December 1999.

Baker, J.: Generation of kilovolt subnanosecond pulses using a nonlinear transmission line, 1993. 